Forum 2019 $\cdot 34: 209$

https://doi.org/10.1007/s12312-019-0568-7

Online publiziert: 14. Februar 2019

C) Springer Medizin Verlag $\mathrm{GmbH}$, ein Teil von Springer Nature 2019

\author{
F. Brandt ${ }^{1} \cdot$ S. Rubai ${ }^{2} \cdot$ C. Keinki ${ }^{3}$ \\ 'IKK Südwest, Projekt PIKKO, Saarbrücken, Deutschland \\ ${ }^{2}$ Saarländische Krebsgesellschaft e.V., Saarbrücken, Deutschland \\ ${ }^{3}$ Deutsche Krebsgesellschaft e.V., Berlin, Deutschland
}

\title{
Patienteninformation, -kommunikation und Kompetenzförderung in der
Onkologie (PIKKO)
} Helfende Hand gegen Krebs

Krebspatienten haben einen hohen Bedarf an Information, Beratung und Unterstützung, der in unserer aktuellen medizinischen Versorgung häufig nicht genug berücksichtigt wird. Mit dem Projekt PIKKO (Patienteninformation, -kommunikation und Kompetenzförderung in der Onkologie) wurde ein neues Instrument geschaffen, um Betroffenen die richtigen Unterstützungsangebote zur richtigen Zeit zur Verfügung zu stellen. PIKKO ist ein Gemeinschaftsprojekt, das von der Deutschen Krebsgesellschaft, der Saarländischen Krebsgesellschaft, dem Universitätsklinikum Jena, der IKK Südwest, der KNAPPSCHAFT und der Techniker Krankenkasse zusammen mit niedergelassenen Haus- und Fachärzten, der Kassenärztlichen Vereinigung Saarland, mehreren saarländischen Kliniken und weiteren starken Partnern im Saarland umgesetzt wird.

Weil Wissen stärkt: Mit einem zusätzlichen Beratungs- und Informationspfad will PIKKO den Betroffenen einer Krebs- erkrankung ermöglichen, eine informierte Therapieentscheidung zu treffen und sich aktiv und selbstbestimmt mit ihrer Erkrankung auseinander zu setzen. Dieser Pfad enthält drei zentrale Elemente:

Betroffene werden von speziell ausgebildeten Onko-Lotsen systematisch mit allen wesentlichen Informationen versorgt und durch die Therapie begleitet. In der spezialisierten onkologischen Beratung, die von der Saarländischen Krebsgesellschaft angeboten wird, werden psychische und soziale Bedürfnisse der Betroffenen berücksichtigt. Zusätzlich wurde durch die Deutsche Krebsgesellschaft die onkologische Wissensdatenbank „Mein PIKKO“ erstellt und als Informationstool in die Versorgung integriert. Sie ermöglicht einen selbständigen und barrierefreien Zugang zu qualitätsgesicherten Informationen rund um die Krebserkrankung.

Das Projekt wird durch den Innovationsfonds beim Gemeinsamen Bundesausschuss gefördert. Zudem wird das
Projekt vom saarländischen Ministerium für Soziales, Gesundheit, Frauen und Familie unterstützt. Eine Teilnahme an dem Projekt ist für Versicherte der teilnehmenden Krankenkassen zunächst bis zum 31.10.2019 möglich. Projektbegleitend erfolgt eine wissenschaftliche Auswertung durch das Institut für Psychosoziale Medizin und Psychotherapie im Universitätsklinikum Jena. Bei einer positiven Bewertung soll das Projekt fortgeführt werden.

Weitere Informationen rund um das Projekt PIKKO finden sich auf der Homepage www.pikko.de. Bei Rückfragen ist das PIKKO-Team unter der Telefonnummer 0681/93696-8239 zu erreichen.

\section{Korrespondenzadresse}

\section{Florian Brandt}

Projekt Pikko, IKK Südwest

Berliner Promenade 1, 66111 Saarbrücken,

Deutschland

florian.brandt@ikk-sw.de 